\title{
Modeling and Simulation of Closed-Loop Supply Chains Considering Economic Efficiency
}

\author{
Yoshitaka Tanimizu, Yusuke Shimizu, Koji Iwamura, and Nobuhiro Sugimura \\ Graduate School of Engineering, Osaka Prefecture University, Japan \\ tanimizu@me.osakafu-u.ac.jp
}

\begin{abstract}
This study proposes a basic model of closed-loop supply chains which includes not only traditional forward supply chains for the generation of products but also reverse supply chains for the reuse of products in consideration of economic efficiency for MTO (Make to Order) companies. The model consists of four model components, i.e., clients, manufacturers, suppliers, and remanufacturers. A remanufacturer is added to the previous model of forward supply chains as a new model component which collects used products from clients and provides reusable parts to manufacturers. Remanufacturers as well as manufacturers and suppliers modify their schedules and negotiate with each other in order to determine suitable prices and delivery times of products. Remanufacturers stimulate clients to discard used products to meet the demand of reusable parts. They can increase the amount of reused products and reduce wastes by creating a balance between supply and demand of reusable parts.
\end{abstract}

Keywords: Closed-loop supply chain, Reverse supply chain, Scheduling, Genetic algorithm, Negotiation.

\section{Introduction}

In recent years, many companies focus on incorporating environmental concerns into their strategic decisions [1]. Green supply chain management (GSCM) has gained increasing attention within both academia and industry [2]. The green supply chain is an approach which seeks to minimize a product or service's ecological footprint. The concept of the GSCM covers all the phases of a product's life cycle, from the extraction of raw materials through the design, production and distribution phases, to the use of products by consumers and their disposal at the end of the product's life cycle including reconditioning, reuse, and recycling of products [1]. Products and materials are returned from customers to suppliers or manufacturers through reverse supply chains in order to be recycled, reused or reconditioned. Gungor and Gupta [3] indicate that effort must be made for environmentally conscious manufacturing and product recovery systems to be profitable so that the incentive for development and planning of these systems continues.

This study proposes a closed-loop supply chain model for parts reuse in consideration of economic efficiency. A closed-loop supply chain includes not only traditional forward supply chains for the generation of products but also reverse supply chains 
for the reuse of products. Figure 1 shows a basic configuration of a closed-loop supply chain. Model components in the reverse supply chain collect used products from customers and provide usable parts to assembly manufacturers or parts suppliers. The components are referred to as remanufacturers in this study. This study also proposes a negotiation protocol which synchronizes the demand of reusable parts and the supply of used products among the model components in the closed-loop supply chain. A lot of used products become waste products in traditional recovery approaches, since the customers may discard products without consideration for reuse of products whenever they want. In the proposed new protocol, remanufacturers create a balance between supply of used products from customers and demand of reusable parts to remanufacturers. When manufacturers require usable parts for generating new products, remanufacturers stimulate customers to discard products for reuse by indicating high required prices for used products to customers. On the other hand, when manufacturers require few parts, remanufacturers indicate low required prices to customers in order to discourage customers from discarding products. Remanufacturers can increase the amount of reused products and reduce waste products.

The reminder of this paper is organized as follows. Section 2 reviews the previous supply chain models for forward supply chains. Section 3 describes a new model and a negotiation protocol for closed-loop supply chains. Finally, Section 4 demonstrates experimental results.

\section{Previous Supply Chain Model}

There are a large number of literatures on a closed-loop supply chain which involves studies on network design problems, product acquisition management, marketingrelated issues, etc. [4]. Design problems of closed-loop supply chain networks involve a high degree of uncertainty associated with quality and quantity of used products. Robust optimization, such as stochastic programming, is commonly used to deal with the uncertainty. However uncontrolled acquisition of used products results in excessive inventory levels or stock-outs due to insufficient used products. Marketingrelated issues include the pricing of remanufactured products. Some researches develop game theory-based models to determine prices of remanufactured products.

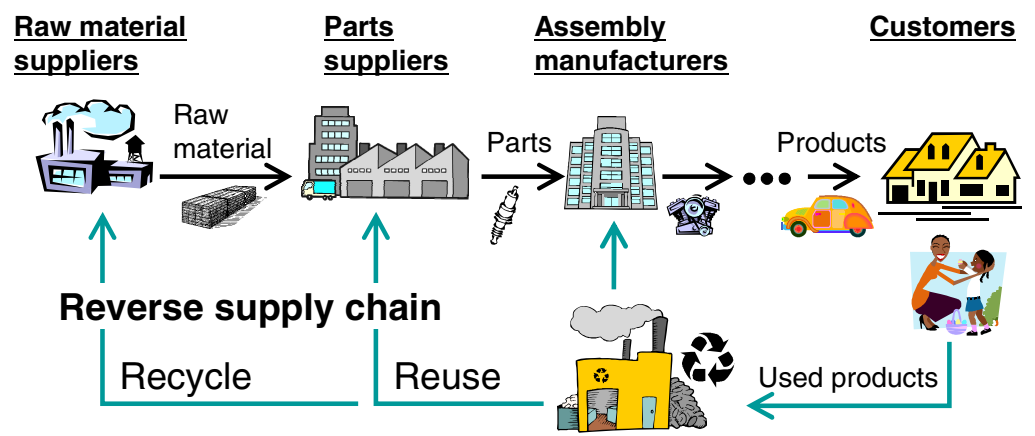

Fig. 1. Closed-loop supply chain 
Our previous studies have represented a framework for dynamically forming and reconfiguring a supply chain as a dynamic supply chain [5]. Each organization in the supply chains can change business partners for every order to find suitable business partners and enter into profitable contracts. A three-layered dynamic supply chain model consisting of clients, manufacturers, and suppliers has been proposed as a minimum model for the multi-layered dynamic supply chains which involve MTO (Make to Order) companies with no inventories [6]. The model provided a negotiation protocol to determine suitable prices and delivery times for ordered products through the iteration of the negotiation process between the organizations, as well as through the modification processes of production schedules.

\section{Closed-Loop Supply Chain Model}

\subsection{Modeling of Remanufacturer}

In this study, a new model component, a remanufacturer, is added to the three-layered model in order to represent the negotiation protocol among organizations in closedloop supply chains. A minimum model for the closed-loop supply chains consists of four components: clients, manufacturers, suppliers, and remanufacturers, as shown in Fig. 2. Remanufacturers have neither stock of usable parts nor used products.

Remanufacturer $R_{s}$ generates an order and sends it to clients in order to collect a used product. The order includes information about the required price $p c r_{s, n}^{O}$ of a used product. The required price is estimated on the required price $p c c_{p, n}^{O}$ of a new product by using the following equation.

$$
p c r_{s, n}^{O}=p c c_{p, n}^{O} \times p r_{s, n}
$$

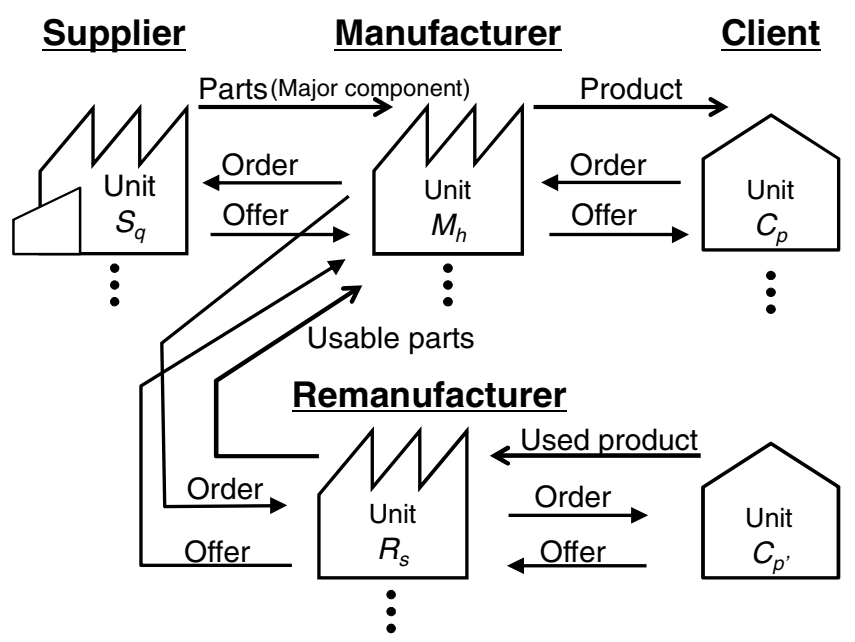

Fig. 2. Closed-loop supply chain model 
where

$-p r_{s, n} \quad$ A factor related to a required price of a used product

The remanufacturer also generates an offer of a usable part. The offer includes the possible delivery time and the bid price of the usable part. The possible delivery time $d t r_{s, h, n}^{F}$ and the bid price $p c r_{s, h, n}^{F}$ are determined as shown in the following equations based on the modified schedule of the remanufacturer.

$$
\begin{gathered}
d t r_{s, h, n}^{F}=c t r_{s, h, n} \\
p c r_{s, h, n}^{F}=t c r_{s, h, n}+r w r_{s, h, n}-p n r_{s, h, n}+\sum_{g=1}^{G} \Delta p n r_{s, g}^{R v(s, n, r)} \\
t c r_{s, h, n}=d c r_{s, h, n}+r c r_{s, h, n}+p p r_{s, h, n} \\
r c r_{s, h, n}=p c c_{p, n}^{O} \times F(t) \times c r_{s, h, n} \\
p p r_{s, h, n}=p c r_{s, n}^{O} \\
p n r_{s, h, n}=k m_{h, n} \times \max \left\{d t r_{s, h, n}^{F}-d t m_{h, n}^{O}, 0\right\}
\end{gathered}
$$

where

$-c t r_{s, h, n}$

$-t c r_{s, h, n}$

- $r w r_{s, h, n}$

- $p n r_{s, h, n}$

- $\Delta p n r_{s, g}^{R v}$

- $d c r_{s, h, n}$

- $r c r_{s, h, n}$

- $p p r_{s, h, n}$

$-F(t)$

$-c r_{s, h, n}$

- $k m_{h, n}$

$-d t m_{h, n}^{O}$
Completion time of a usable part generated by remanufacturer $R_{s}$ Total cost of a usable part

Reward for a usable part required by remanufacturer $R_{s}$

Penalty charge due to delay of a usable part

Penalty charge due to delay in delivery time of contracted orders

Disassembly cost of a usable part

Repair cost of a usable part

Purchase price of an used product

Cumulative failure rate estimated based on the Weibull distribution

A factor related to a repair cost of a usable part

Penalty charge factor representing penalty charge per unit time

Required delivery time of a usable part for product $N C_{p, n}$.

\subsection{Extension of Client Model}

A client provides a new function to determine discarding a used product in this study. The client decides when to discard a used product by using the following equation.

$$
u t c_{p^{\prime}, n^{\prime}} \geq p l c_{p^{\prime}, n^{\prime}}
$$


where
- $u t c_{p^{\prime}, n^{\prime}}$
A period of time when a product has been used by client $C_{p}$,
- $p l c_{p^{\prime}, n^{\prime}}$
Product's life cycle estimated based on the Weibull distribution

In general, a client independently discards a product which has been used beyond its life cycle. The product is dealt with as a waste, if it is not required to be reused.

In the proposed model, a client firstly receives a requirement for reuse of a product from a remanufacturer as an order. The client receiving the order evaluates a motivation for discarding a product by using the following equations and determines which product is discarded. If some products satisfy the condition determined by Eq. (9), the client determines discarding a product which has the highest value of the motivation in Eq. (10).

$$
\begin{gathered}
m v_{p^{\prime}, n^{\prime}} \geq r n \\
m v_{p^{\prime}, n^{\prime}}=\frac{p c r_{s, n}^{O}-\left(p v_{p^{\prime}, n^{\prime}}-d c c_{p^{\prime}, n^{\prime}}\right)}{p c r_{s, n}^{O}}
\end{gathered}
$$

where

$\begin{array}{ll}-m v_{p^{\prime}, n^{\prime}} & \text { Motivation for providing a product to remanufacturers for reuse } \\ -r n & \text { Random numbers } \\ -p v_{p^{\prime}, n^{\prime}} & \text { Product's value which decreases with time of use of a product from } \\ & \text { the initial price. It is estimated based on the Weibull distribution. } \\ -d c c_{p^{\prime}, n^{\prime}} & \text { Cost for discarding a product by client } C_{p^{\prime}}\end{array}$

\subsection{Negotiation Protocol}

In a conventional model, a client firstly determines which products should be discarded by using Eq. (8), regardless of the requirements from remanufacturers. Remanufacturers can only reuse the products discarded by the client. When no remanufacturers require the discarded products during a certain period of time, they must be waste products.

The proposed closed-loop supply chain model creates a balance between supply of used products from customers and demand of reusable parts for manufacturers. A remanufacture stimulates the intention of the client to discard a used product by providing the higher required price $\operatorname{prr}_{s, n}^{O}$, when the product is required for reuse. The client throws out the product easily, even if the product is not yet up to its life cycle described in Eq. (8). The remanufacturer deals with a client as a virtual warehouse. The amount of waste products decreases and a lot of used products are reused in the reverse supply chains.

Steps in the negotiation process in the closed-loop supply chains are as follows:

1. Manufacturer $M_{h}$ sends a new order for a part to all suppliers and remanufacturers.

2. Remanufacturer $R_{s}$ creates a new order for a used product and sends it to all clients. 
3. Client $C_{p}$, specifies candidates for discarding products by using Eq. (8), and generates an offer of the used product which is selected by Eqs. (9) and (10).

4. Remanufacturer $R_{s}$ improves a schedule by using a genetic algorithm (GA) after adding disassembly processes and repair processes of the used product. Then, the remanufacturer creates an offer for a usable part and sends it to manufacturer $M_{h}$.

5. Manufacturer $M_{h}$ selects one offer which has the lowest bid price. Then, the manufacturer improves a production schedule by using a GA after adding manufacturing processes of the selected part and generates an offer for client $C_{p}$.

This negotiation process is repeated among the organizations in the closed-loop supply chains until the client accepts an offer from a manufacturer or cancels the order.

\section{Computational Experiments}

\subsection{Experiments for Comparing Proposed Model with Conventional Model}

A prototype of a simulation system for closed-loop supply chains has been developed using Windows-based networked computers (Intel Core 2 Duo E8500 3.16 GHz CPU with 1.99 GB of RAM). Two suppliers, two manufacturers, a remanufacturer, and a client were implemented as agents on different six computers, as shown in Fig. 3.

In the initial conditions, the suppliers and the manufacturers had same job-shop type production schedules consisting of 5 resources and 20 contracted parts, and 10 resources and 20 contracted products, respectively [6]. The client continuously generated 100 new orders and negotiated with two manufacturers during the experiments

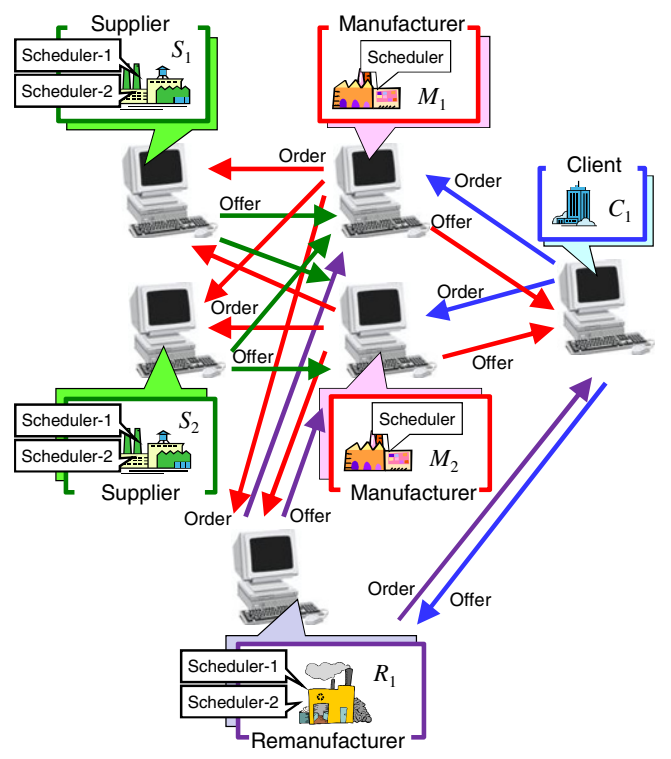

Fig. 3. Prototype of simulation system 
for about 6 hours. The manufacturers generated orders and negotiated among the two suppliers and one remanufacturer every 30 seconds of bidding time in order to generate offers for the client. Population size, crossover rate, and mutation rate of the GA were $30,0.8$, and 0.2 , respectively.

Ten experiments were carried out with the proposed model. The values of parameters $p r_{s, n}$ and $c r_{s, n}$ used in Eqs. (1) and (5) was set to 0.15 and 0.01, respectively. The experimental results are summarized in Table 1.

The experimental results were compared with the ones of a conventional model in which the client independently determines when to discard products. Ten experiments of the conventional model were also carried out on the same experimental conditions of the proposed method. The experimental results of the conventional model are summarized in Table 1. The remanufacturer of the proposed model can increase the rate of reused parts about $47 \%$ more than the one of the conventional model.

Table 1. Comparison of experimental results

\begin{tabular}{llllll}
\hline [average] & $\begin{array}{l}\text { Num. of } \\
\text { Reused } \\
\text { products }\end{array}$ & $\begin{array}{l}\text { Num. of } \\
\text { Waste } \\
\text { products }\end{array}$ & $\begin{array}{l}\text { Rate of } \\
\text { reuse to } \\
\text { waste }\end{array}$ & $\begin{array}{l}\text { Rate of } \\
\text { reused parts } \\
\text { to new parts }\end{array}$ & $\begin{array}{l}\text { Profit of } \\
\text { remanufacturer } \\
\left(* 10^{3} \$\right)\end{array}$ \\
\hline $\begin{array}{l}\text { Proposed } \\
\text { model }\end{array}$ & 43.2 & 37.7 & $53.4 \%$ & $54.6 \%$ & 89.7 \\
\hline $\begin{array}{l}\text { Conventional } \\
\text { model }\end{array}$ & 5.7 & 50.2 & $10.2 \%$ & $7.8 \%$ & 40.7 \\
\hline
\end{tabular}

\subsection{Simulation for Performance Measurement}

This prototype of a simulation system can be used for the organizations in the closedloop supply chains to make effective plans for reuse of products in consideration of economic efficiency. In this paper, about 100 experiments were carried out on

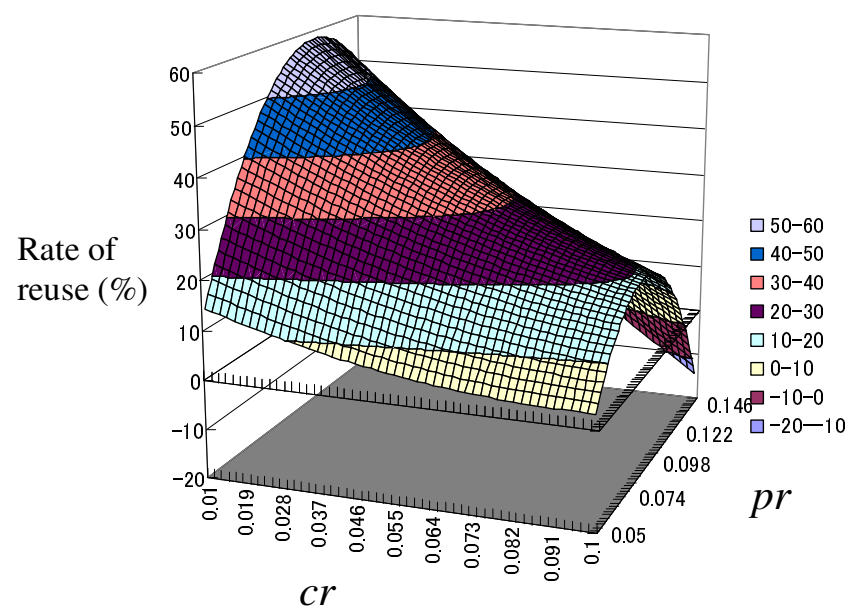

Fig. 4. Performance measurement of closed-loop supply chains 
25 experimental conditions by changing the values of parameters $c r_{s, n}$ and $p r_{s, n}$ which was used to determine a repair cost of a usable part and a required price of a used product, respectively. Figure 4 describes the least squares approximation of the rate of reuse which has been derived from the experimental results. As shown in this figure, the parts with higher repair costs decrease the rate of reuse. Not only the used products purchased at low prices but also the ones purchased at high prices decrease the rate of reuse, since the client cannot provide the used products at lower prices than the product's value and the manufacturers cannot enter into a contract with the remanufacturer at higher price than the price which the manufacturer requires.

\section{Conclusion}

This study proposes a closed-loop supply chain model consisting of suppliers, manufacturers, remanufacturers, and clients, as a minimum model for the closed-loop supply chains. It also proposes a negotiation protocol among the organizations in the closed-loop supply chains. A prototype of a simulation system was developed to evaluate the effectiveness of the model and protocol. The proposed model was compared with a conventional model which discarded the used products without negotiation processes between remanufacturers and clients. The experimental results show that the proposed model can reuse products more than the conventional model. The prototype system can be used for performance measurement of closed-loop supply chains.

\section{References}

1. BearingPoint: 2008 Supply Chain Monitor “How Mature is the Green Supply Chain?”. Survey report (2008)

2. Sarkis, J., Zhu, Q., Lai, K.: An Organizational Theoretic Review of Green Supply Chain. International Journal of Production Economics 130, 1-15 (2011)

3. Gungor, A., Gupta, S.M.: Issues in Environmentally Conscious Manufacturing and Product Recovery: a Survey. Computers and Industrial Engineering 36, 811-853 (1999)

4. Llgin, M.A., Gupta, S.M.: Environmentally Conscious Manufacturing and Product Recovery (ECMPRO): A Review of the State of the Art. Journal of Environmental Management 91, 563-591 (2010)

5. Tanimizu, Y., Yamanaka, M., Iwamura, K., Sugimura, N.: Multi-Agent Based Dynamic Supply Chain Configuration Considering Production Schedules. In: Proc. of International Symposium on Flexible Automation, pp. 572-578 (2006)

6. Tanimizu, Y., Ozawa, C., Shimizu, Y., Orita, B., Iwamura, K., Sugimura, N.: Flexible Multi-Layered Dynamic Supply Chain Models with Cooperative Negotiation. International Journal of Automation Technology 7(1), 128-135 (2013)

7. Lawrence, S.: Resource Constrained Project Scheduling: An Experimental Investigation of Heuristic Scheduling Techniques (Supplement), Graduate School of Industrial Administration, Carnegie-Mellon University, Pittsburgh, Pennsylvania (1984) 